\title{
Ventricle Registration for Inter-subject White Matter Lesion Analysis
}

\author{
Cynthia Jongen, Jeroen van der Grond, and Josien P.W. Pluim \\ Image Sciences Institute, University Medical Center Utrecht, Heidelberglaan 100, E01.335, \\ Utrecht, The Netherlands \\ \{cynthia, jeroen, josien\}@isi.uu.nl
}

\begin{abstract}
A method is presented to non-rigidly register lateral ventricles to enable the automated analysis of peri-ventricular white matter lesions. A binary average image of the lateral ventricle system is used as a reference image for registration. To prevent false deformations of the lesions we non-rigidly register CSF segmentations to the average lateral ventricle image. The subvoxel accuracy achieved, allows accurate mapping of the peri-ventricular white matter lesions to the reference space of the average lateral ventricles. Application to patient data shows the feasibility of the presented approach.
\end{abstract}

\section{Introduction}

White matter lesions show as hyperintensities on T2-w and FLAIR MR images. These hyperintense white matter lesions are associated with age, vascular risk factors, such as hypertension and diabetes mellitus, and clinically silent stroke $[1,2,3,4]$. Analysis of white matter lesions has mainly focused on comparing lesion severity by grading the amount of lesion or volume measurements of segmented lesions. Analysis of differences in lesion location has been limited to distinguishing deep white matter lesions from peri-ventricular white matter lesions [4] and grading lesion severity for a small number of peri-ventricular areas [1,2].

The goal of this work is to analyze differences in lesion location between diabetes patients and controls on a voxel-by-voxel basis, without needing to classify lesions into a limited number of categories. To allow the analysis, MR images of different patients need to be matched. Since our interest is focused on peri-ventricular white matter lesions it is important that the ventricular system is correctly matched. Furthermore, matching should not actively deform the white matter lesions. Since the shape of the ventricular system has a high inter-subject variability, fine scale nonrigid deformations are necessary. If gray-value images are used, these fine scale deformations will also deform the lesions by matching lesion to lesion or lesion to healthy tissue. Of course this could be avoided by masking the lesions, but this approach will affect patients with different degrees of lesion severity differently. Therefore, we have chosen not to use the gray-value images for registration, but to match binary CSF segmentations of patients and controls to a binary reference lateral ventricle system image. Since peri-ventricular white matter lesions adjoin the lateral ventricles, any local inter-subject differences will be largely given by the differences between ventricular systems. So, deforming the ventricle systems to match with a 
reference lateral ventricle image will also register the peri-ventricular white matter lesions. We could also have included CSF around the cortex in our reference image, but the gyri and sulci pattern is highly variable between subjects. Therefore, mismatches of the CSF in different sulci are likely. This might adversely affect the registration of the lateral ventricles and thereby the peri-ventricular lesions. Even if a correct matching of CSF in the sulci is achieved, the beneficial effect on registration accuracy for the lesions will be small. No other structures that could easily be used for registration are present near the ventricles.

Thus, our approach of registering CSF segmentations to a binary average lateral ventricle image and applying these deformations to the lesions, allows us to place the lesions of different subjects in the same reference space. This enables us to compare the lesion pattern in patients to the lesion pattern in control subjects.

\section{Methods}

\subsection{MR Imaging}

Brain MR images were acquired on a Philips Gyroscan ACS-NT 15 whole body system operating at 1.5 Tesla (Philips Medical Systems, Best, The Netherlands). All patients were scanned using the same MR protocol consisting of T1-w, IR, T2-w, PD and FLAIR scans. All scans had a slice thickness of $4 \mathrm{~mm}, 38$ continuous slices, a 230 x $230 \mathrm{~mm}$ field of view, and a 256 x 256 scan matrix. The scan parameters were: T1w (FFE): 234/2 ms (repetition time (TR)/echo time (TE)); IR: 2919/410/22 ms (TR/inversion time (TI)/TE); T2-w: 2200/100 ms (TR/TE); PD: 2200/11 ms (TR/TE); and FLAIR: 6000/2000/100 ms (TR/TI/TE).

\subsection{Image Preprocessing}

The image preprocessing consisted of three steps. First, an automatic correction of shading artifacts in the T1-w, T2-w, PD, and FLAIR images was done. The intensity distortions were modeled with a multiplicative fourth order polynomial, as described by Likar et al. [5].

Second, for each patient and control the shading corrected T2-w, PD, FLAIR, and the IR images were registered to the shading corrected T1-w image using a normalized mutual information based registration algorithm [6] optimizing 9 parameters (translation, rotation, and scaling).

Third, CSF was extracted by k-means clustering of the 5 registered MR images into 8 clusters. Initialization of the cluster centers was done by clustering 10 random subsamples of $0.1 \%$ of all data using a random cluster center initialization and smoothing the result by clustering the centers found for the subsamples [7]. The CSF cluster was manually selected from the resulting clustered image. Making a distinction between lateral ventricle and other CSF structures would require much user interaction and the presence of non-ventricle structures was not expected to make a substantial difference to the registration results. Therefore, after interpolating the slices to isotropic voxels of $0.9 \mathrm{~mm}^{3}$ using shape-based interpolation [8], the whole CSF cluster was used for registration to an average ventricle shape. 


\subsection{Construction of Average Ventricle}

An average ventricle is needed as a template for inter-subject registration of CSF segmentations. Images of 24 patients with arterial vascular disease, who were not included in this study, were used. These patients are similar in age and in changes of the ventricle shape as the control and diabetics subjects. The lateral ventricles were segmented by thresholding and manual editing of their FLAIR scans (same protocol as used in this study). This resulted in 24 binary lateral ventricle segmentations. The slices of the segmentation images were interpolated to isotropic voxels using shapebased interpolation [8]. Eight segmented ventricles were registered together to determine their average ventricle position and size using a 12 parameter affine registration algorithm [9]. The eight ventricles were transformed to this position, summed, and thresholded. Then, this image was used as template for the affine registration of all 24 segmented ventricles. The 24 affinely registered images were summed. Next, a Bspline based non-rigid registration algorithm [9] was used with a control point spacing (CPS) of 32 voxels to register all 24 affinely registered ventricles to the sum image. A large CPS was chosen because we only want to correct for global differences in ventricle shape. The non-rigidly registered ventricles were summed and thresholded keeping the variation in ventricle shape that was present in at least a third of the ventricles. The resulting image was used as reference image for the affine and non-rigid registration of CSF segmentations of patients and controls.

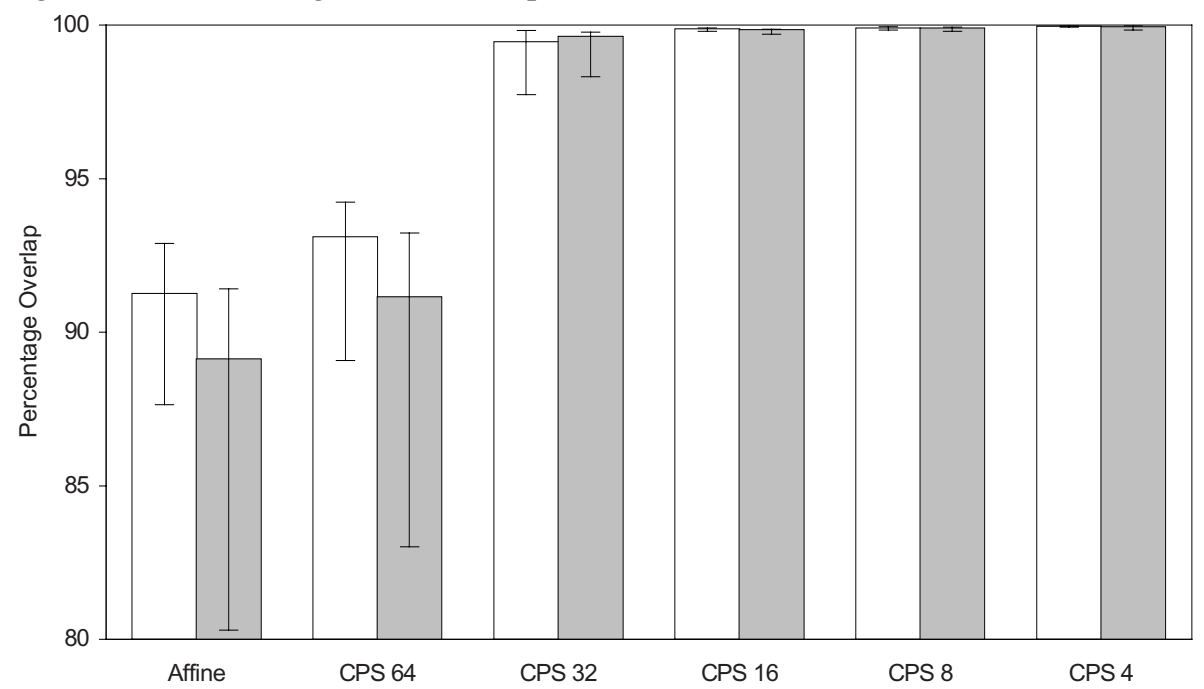

Fig. 1. Median percentage of average ventricle overlapped by CSF of diabetes patients (white) and control subjects (gray) after registration with an increasing degree of non-rigidity. Error bars indicate 25 and 75 percentiles

\subsection{Registration}

Registration of the CSF segmentations to the average ventricle shape was done using the method described by Rueckert et al. [9]. This method proceeds in two steps. First, 
a 15 parameter affine registration using normalized mutual information $[6,10]$ was done to correct for global differences in position and size. Next, a free-form deformation using a B-spline based non-rigid registration algorithm with normalized mutual information as registration criterion was done. The spacing of the control points was decreased from 64 to 4 voxels by factors of 2 .

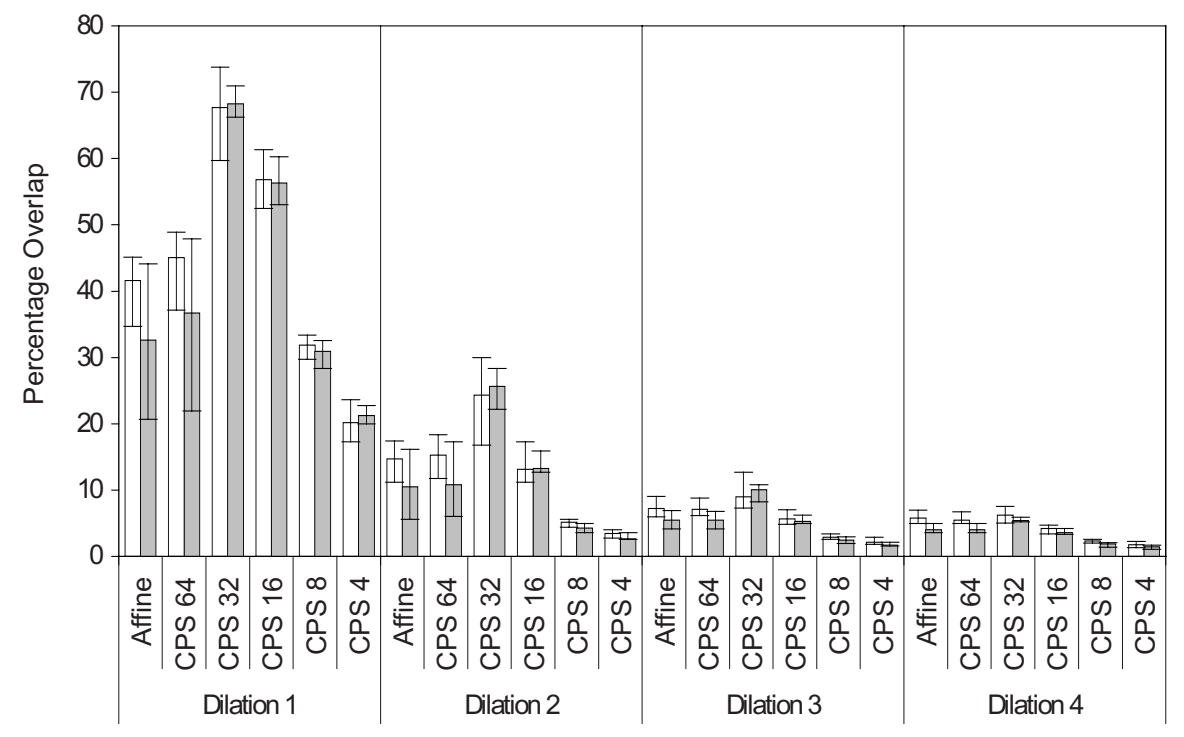

Fig. 2. Median percentage of overlap of rings of one voxel dilations around the average ventricle with CSF segmentations of diabetes patients (white) and control subjects ( gray) after registration with an increasing degree of non-rigidity. Error bars indicate 25 and 75 percentiles

\subsection{Statistical Analysis of White Matter Lesion Patterns}

To illustrate the proposed method, we applied it to twenty-four diabetes mellitus patients and twelve control subjects matched for age. White matter lesion segmentations were made using the method described in [11]. All white matter lesion segmentations were transformed to the reference average ventricle shape by applying the transformations found for the CSF segmentation registration. Differences in white matter lesion patterns between diabetics and controls were analyzed using the non-parametric statistical analysis described by Holmes et al. [12]. This test is based on generating randomizations of the distribution of observations across the control and the diabetics group. The test statistics resulting from the randomizations were compared with the test statistic resulting from the experimental observation. Thus, the probability distribution of the test statistic was calculated based on the experimental data. This approach allowed us to calculate for each voxel the probability that the control and diabetics group were drawn from the same population. 


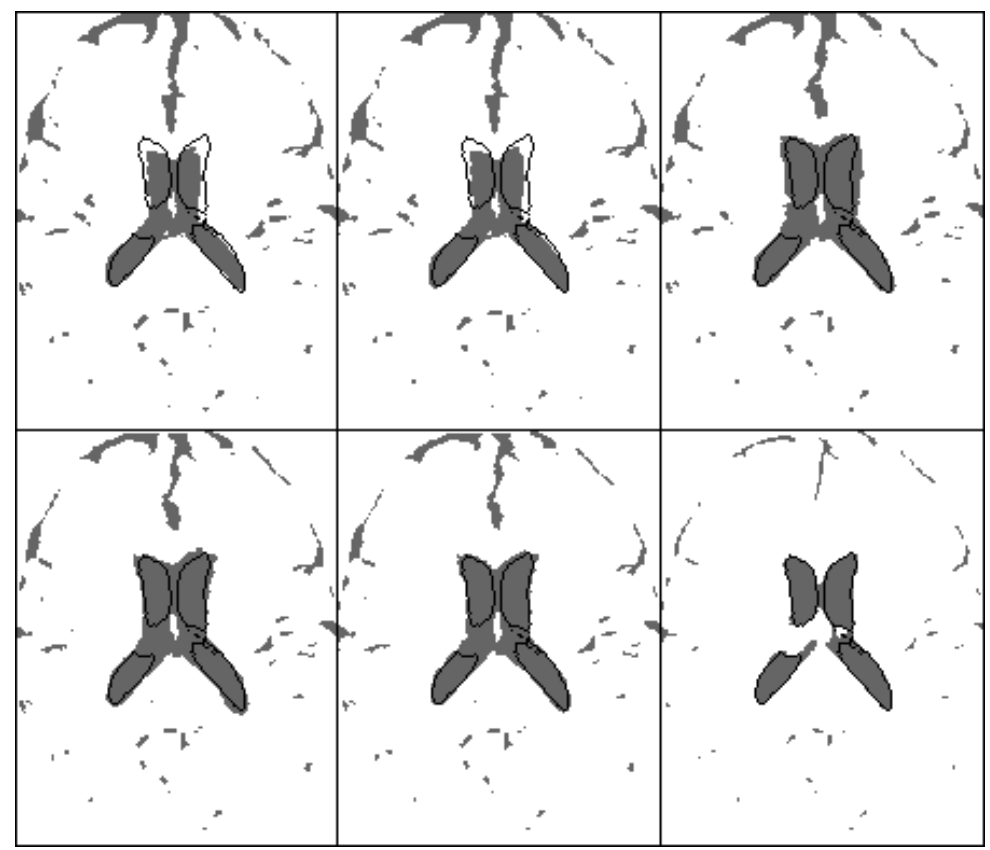

Fig. 3. Registration of the CSF segmentation of a subject (gray) to the average ventricle (black lines). The top row shows from left to right the result after registration using affine transformations, CPS 64 and CPS 32 non-rigid registration. The bottom row shows from left to right the result after non-rigid registration using CPS 16, CPS 8, and CPS 4

\section{Results}

\subsection{Registration}

Usually, the overlap between two segmentations is calculated as a measure of registration accuracy by taking two times the number of overlapping voxels divided by the sum of the total number of voxels in both segmentations. Since in this case one segmentation contains all CSF structures and the other only lateral ventricles, this would not yield relevant numbers. Therefore, we have constructed a one-voxel dilation around the average ventricles. Next, we constructed a one-voxel dilation around this first dilation, then around the second and around the third. So, we have four one voxel $(0.9 \mathrm{~mm})$ thick structures (referred to as dilation 1 (closest to the average ventricles) to 4 (farthest away)) and for each we counted the number of CSF segmentation voxels overlapping with this structure.

Figure 1 shows the percentage of average ventricle voxels that are overlapped by the CSF segmentation of diabetes patients and controls for the subsequent steps in the registration procedure. In Figure 2 the overlap of the CSF segmentation with the onevoxel dilations around the average ventricles is shown. Ideally, the overlap with the average ventricles is a hundred percent and the overlap with the dilations is zero. 


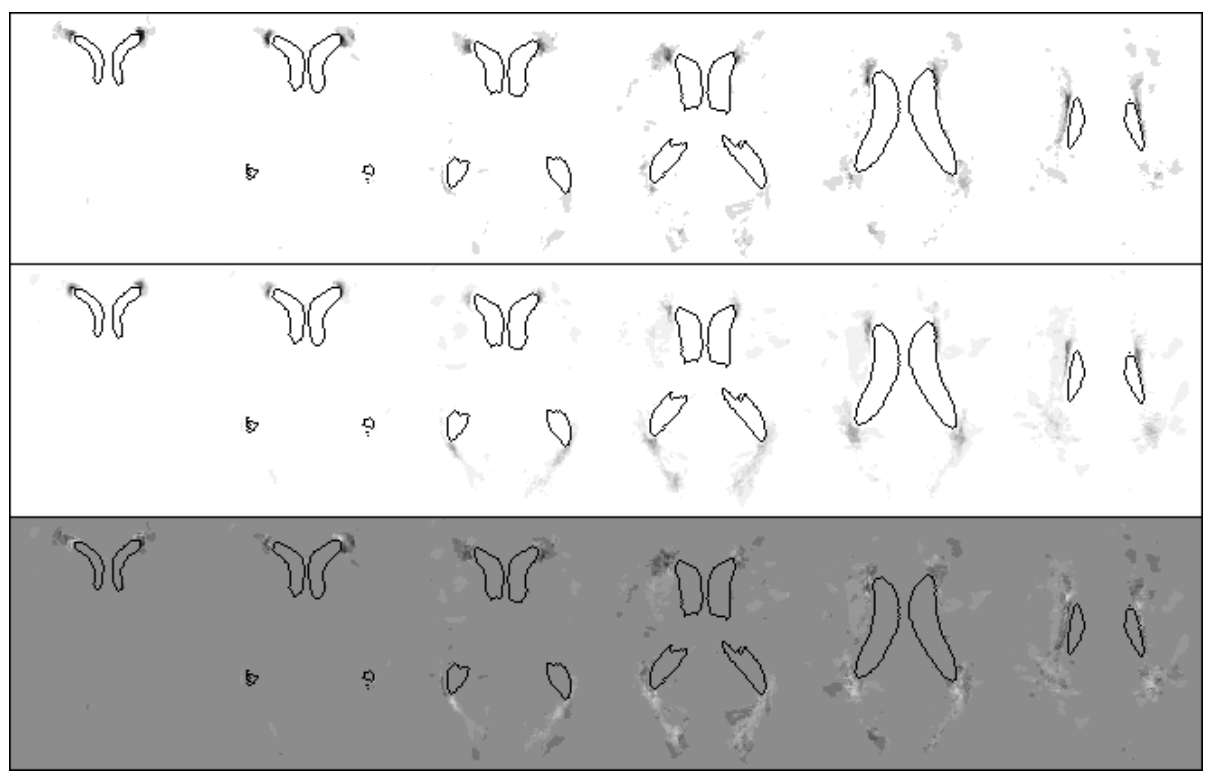

Fig. 4. Lesion pattern image for control subjects (top row) and diabetes patients (middle row). Darker gray indicates a higher prevalence of lesion. The bottom row shows the difference in lesion patterns with whiter areas indicating a higher lesion prevalence in diabetes patients and darker areas a higher prevalence in control subjects. The black lines show the outline of the average lateral ventricle.

After registration with a control point spacing of 32 voxels, almost all voxels of the average ventricle are overlapped by voxels of the CSF segmentations. However, this is at the expense of the overlap with the dilations, which is also increased. Thus, the CSF segmentation has deformed so that its ventricles have become somewhat larger than the average ventricles. Refinement of the control point grid decreases the overlap with the dilations while retaining and even slightly improving the overlap with the average ventricles.

For finer CPSs the overlap with the dilations is limited to the first dilation. The overlap in dilations two, three and four, especially for CPS eight and four, is caused by the presence of structures in the CSF segmentations other than lateral ventricles such as the third and fourth ventricle.

In Figure 3 the registration of a patient to the average ventricle is shown. Note that the CSF structures not present in the average ventricle image are subject to shrinkage. Inspection of the deformation fields reveals that this is only a local effect, which does not influence the deformations in the peri-ventricular areas.

\subsection{White Matter Lesion Patterns}

Figure 4 shows the lesion pattern in diabetes patients and control subjects, corrected for group size, and a subtraction image showing the differences between patients and controls. The lesion patterns did not differ significantly between diabetes patients and controls. 


\section{Discussion}

We developed an automatic approach to inter-subject ventricle registration for the analysis of peri-ventricular white matter lesions. We constructed a binary average ventricle image as reference to match segmented CSF from different subjects. The registrations of the CSF to the lateral ventricles were accurate, mostly within one dilation of $0.9 \mathrm{~mm}$. The approach of registering all CSF to only lateral ventricles did not lead to inaccuracies in the peri-ventricular regions.

The lesion patterns presented are preliminary and only intended to demonstrate the suitability of the presented method. The number of subjects is too small to have clinical implications. It is clear, however, that our approach provides a way to gain insight into the specifics of the lesion pattern of a patient group. In conclusion, the presented approach of registering CSF to an average ventricle is accurate, feasible, and well suited for the analysis of peri-ventricular white matter lesions.

Acknowledgements. The authors would like to thank the following people and laboratories for kindly supplying them with software and data:

- The Laboratory for Medical Imaging Research in Leuven for the software for mutual information-based registration

- B. Likar from the Department of Electrical Engineering of the University of Ljubljana for the software for MR intensity inhomogeneity correction

- D. Rueckert from the Computational Imaging Science Group, King's College London for the software for non-rigid registration using free-form deformations

- The Utrecht Diabetic Encephalopathy Study group of the University Medical Center Utrecht for the image data.

\section{References}

1. de Leeuw, F.E., de Groot, J.C., Achten, E., Oudkerk, M., Ramos, L.M., Heijboer, R., Hofman, A., Jolles, J., van Gijn, J., Breteler, M.M.: Prevalence of cerebral white matter lesions in elderly people: a population based magnetic resonance imaging study. The Rotterdam Scan Study. J. Neurol. Neurosurg. Psychiatry 70 (2001) 9-14

2. de Leeuw, F.E., de Groot, J.C., Oudkerk, M., Witteman, J.C., Hofman, A., van Gijn, J., Breteler, M.M.: Hypertension and cerebral white matter lesions in a prospective cohort study. Brain 125 (2002) 765-772

3. Longstreth, W.T., Jr., Manolio, T.A., Arnold, A., Burke, G.L., Bryan, N., Jungreis, C.A., Enright, P.L., O'Leary, D., Fried, L.: Clinical correlates of white matter findings on cranial magnetic resonance imaging of 3301 elderly people. The Cardiovascular Health Study. Stroke 27 (1996) 1274-1282

4. Taylor, W.D., MacFall, J.R., Provenzale, J.M., Payne, M.E. , McQuoid, D.R., Steffens, D.C., Krishnan, K.R.: Serial MR imaging of volumes of hyperintense white matter lesions in elderly patients: correlation with vascular risk factors. AJR Am. J. Roentgenol. 181 (2003) 571-576

5. Likar, B., Viergever, M.A., Pernus, F.: Retrospective correction of MR intensity inhomogeneity by information minimization. IEEE Trans Med Imaging 20 (2001) 1398-1410 
6. Maes, F., Collignon, A., Vandermeulen, D., Marchal, G., Suetens, P.: Multimodality image registration by maximization of mutual information. IEEE Trans Med Imaging 16 (1997) 187- 198

7. Bradley, P.S., Fayyad, U.M.: Refining initial points for K-means clustering. International Conference on Machine Learning (1998) 91-99

8. Herman, G.T., Zheng, J.S., Bucholtz, C.A.: Shape-based interpolation. IEEE Computer Graphics and Applications 12 (1992) 69-79

9. Rueckert, D., Sonoda, L.I., Hayes, C., Hill, D.L.G., Leach, M.O., Hawkes, D.J.: Nonrigid registration using free-form deformations: application to breast MR images. IEEE Trans Med Imaging 18 (1999) 712-721

10. Studholme, C., Hill, D.L.G., Hawkes, D.J.: An overlap invariant entropy measure of 3D medical image alignment. Pattern Recognition 32 (1999) 71-86

11. Anbeek, P., Vincken, K.L., van Osch, M.J.P., Bisschops, R.H.C., van der Grond, J.: Probabilistic segmentation of white matter lesions in MR imaging. Neuroimage in press (2004)

12. Holmes, A.P., Blair, R.C., Watson, J.D.G., Ford, I.: Nonparametric analysis of statistic images from functional mapping experiments. J Cereb Blood Flow Metab 16 (1996) 7-22 